Corrigendum

\title{
Corrigendum to "Fibrinogen as a Prognostic Predictor in Pediatric Patients with Sepsis: A Database Study"
}

\author{
Xiaomeng Tang, ${ }^{1,2}$ Lujing Shao, ${ }^{1,2}$ Jiaying Dou, ${ }^{1}$ Yiping Zhou, ${ }^{1}$ Min Chen, ${ }^{3}$ Yun Cui, ${ }^{1}$ \\ Yucai Zhang $\mathbb{1},{ }^{1,2}$ and Chunxia Wang $\mathbb{\circledR}^{1,2}$ \\ ${ }^{1}$ Department of Critical Care Medicine, Shanghai Children's Hospital, Shanghai Jiao Tong University, Shanghai 200062, China \\ ${ }^{2}$ Institute of Pediatric Critical Care, Shanghai Jiao Tong University, Shanghai 200062, China \\ ${ }^{3}$ Department of Information Technology, Shanghai Children's Hospital, Shanghai Jiao Tong University, Shanghai 200062, China \\ Correspondence should be addressed to Chunxia Wang; karencx0465@163.com \\ Received 9 February 2021; Accepted 9 February 2021; Published 19 February 2021 \\ Copyright ( 92021 Xiaomeng Tang et al. This is an open access article distributed under the Creative Commons Attribution License, \\ which permits unrestricted use, distribution, and reproduction in any medium, provided the original work is properly cited.
}

In the article titled "Fibrinogen as a Prognostic Predictor in Pediatric Patients with Sepsis: A Database Study" [1], there was an error in the abstract where the following values (0.616 [95\% CI: $0.457-0.829], P=0.001 ; 1.397$ [95\% CI: $1.245-1.569], P<0.001$, respectively) should be corrected to (0.619 [95\% CI: $0.435-0.880], P=0.008 ; 1.274$ [95\% CI: 1.126-1.442], $P<0.001$, respectively).

The author explained that the error was introduced during manuscript preparation and confirms that this does not affect the results and conclusions of the article.

\section{References}

[1] X. Tang, L. Shao, J. Dou et al., "Fibrinogen as a Prognostic Predictor in Pediatric Patients with Sepsis: A Database Study," Mediators of Inflammation, vol. 2020, Article ID 9153620, 10 pages, 2020. 